\title{
Hausdienst/Liegenschaft
}

\author{
Tätigkeitsbericht 2002
}

Erika Flückiger

Am Donnerstag, den 14. März 1974, wurde das Bauvorhaben: «Abbruch der Gebäude Elfenstrasse 18, 18A und 18B und Erstellen eines Ärzte-Bürogebäudes mit unterirdischer Autoeinstellhalle für $21 \mathrm{PW}$ im Anzeiger für die Stadt Bern publiziert. Ebenfalls im März 1974 wurde der neue Nutzungszonenplan im Entwurf publiziert. Er war aufgrund einer im Herbst 1972 vom Stadtrat mit grosser Mehrheit überwiesenen Motion ausgearbeitet worden. Der Plan sollte vor allem die bedrohliche Zunahme von Büros und andern Arbeitsplätzen in den zentrumsnahen Quartieren bremsen und den vorhandenen Wohnraum nach Möglichkeit erhalten und mehren. Das Ärztehaus, der Sitz des FMHGeneralsekretariates, liegt im Berner Brunnadernquartier, umgeben von Botschaften und Konsulaten (Abb. 2). Die Auflagen des Nutzungszonenplanes mussten beim Bau berücksichtigt werden, so dass im dritten Stock des Gebäudes zwei grosse Attikawohnungen einge- richtet wurden. Eine dritte Wohnung wurde von allem Anfang an für den Hauswart vorgesehen. Im Erläuterungsbericht zum Baugesuch steht u. a.: «Das Projekt charakterisiert die Eingliederung in das Villen- und Diplomatenquartier. Die Konzeption ist nicht starr als Bürobau, vielmehr ökonomisch und human als Ärztehaus. Die Ästhetik ist kongruent mit den aufwendigen Nachbarbauten. Die Umgebung ist unter Beibehaltung des Grünzuges und Baumbestandes optimal gewahrt. Grundriss und Statik sind auf einem Rastersystem ausgebaut. Bei Verwendung von mobilen Trennwänden sind jederzeit Umdispositionen möglich.» (Abb. 3)

Von letzterem haben wir in den vergangenen Jahren bei hausinternen Büroumzügen, etwa für die Schaffung von mehr Büroraum, bei Umstrukturierungen von Abteilungen usw., mehrmals Gebrauch gemacht. Die Tatsache, dass die Wände ohne grossen Aufwand verschoben werden können, hat aber zuweilen auch relativ rasch

Abbildung 1

Dorinda und Manuel Gonzalez.

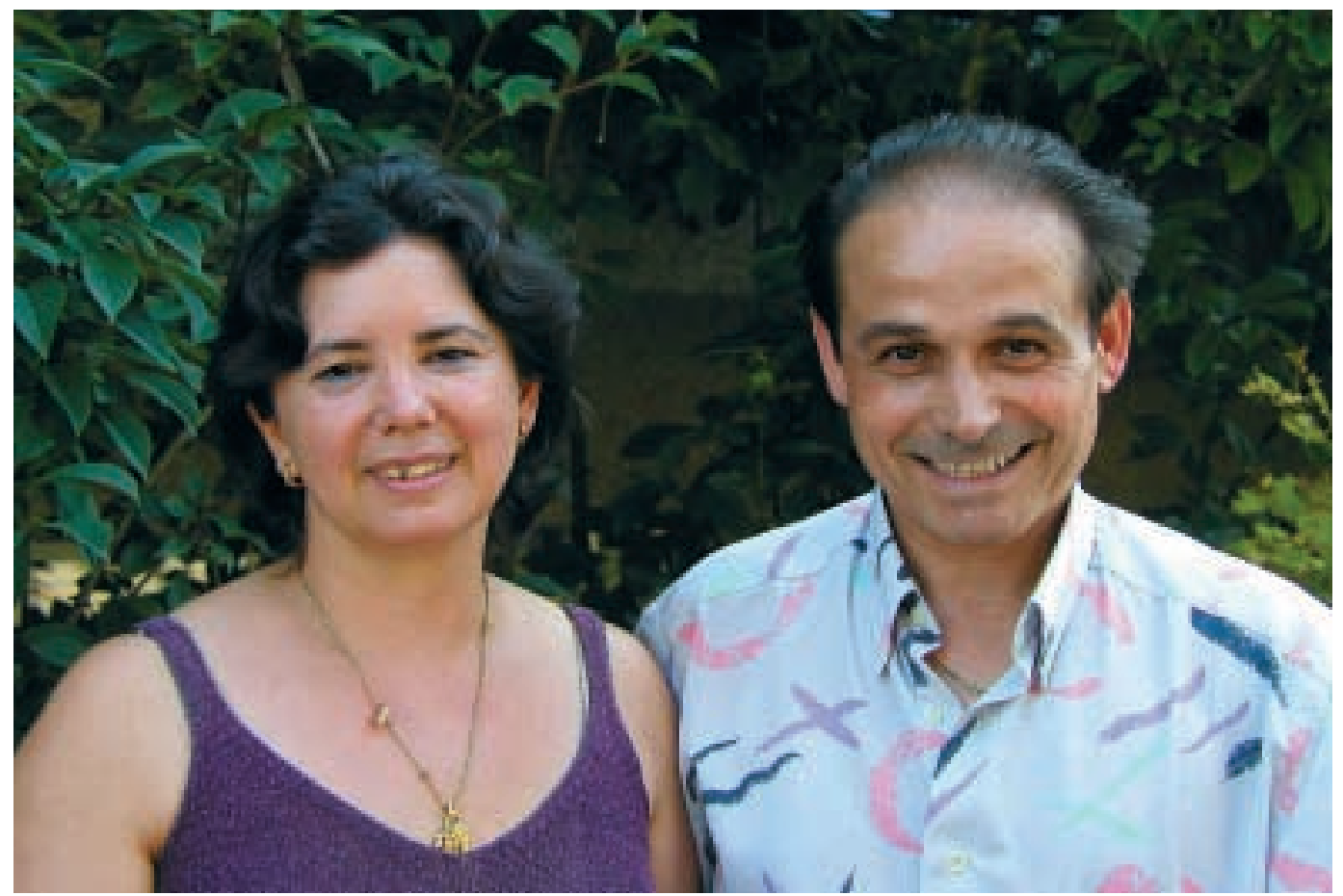


Abbildung 3

FMH-Generalsekretariat, Nord- und Ostseite.

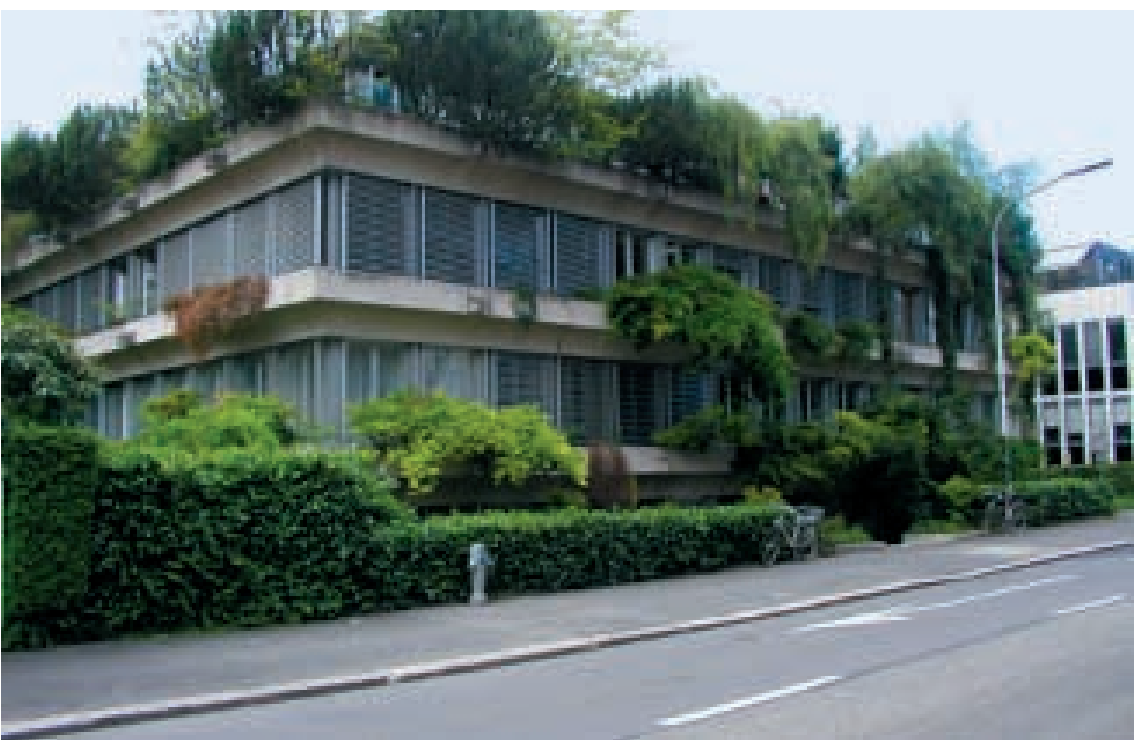

Gelüste geweckt. In der heutigen Konstellation umfasst das FMH-Generalsekretariat im Erdgeschoss ein grosses mit Hellraumprojektor, fix installiertem Beamer, Flipchart, Fernseh- und Videogerät und genügend Stromanschlüssen für Notebooks modern eingerichtetes Sitzungszimmer, das vom Zentralvorstand, von den FMHKommissionen und Abteilungen des Generalsekretariates genutzt wird, eine Cafeteria, in der sich Mitarbeiterinnen und Mitarbeiter während der Kaffee- und Mittagspausen aufhalten und verpflegen und wo z. B. auch der ZV sein Mittagessen einnimmt, den Empfang/Telefonzentrale sowie 7 Büros, die von der Abteilung Aus-, Weiterund Fortbildung (AWF) belegt sind. Der erste Stock umfasst 14 Büros und die Wohnung des Hauswartes. Im zweiten Stock schliesslich befinden sich 16 Büros, eines davon ist der Computerraum mit unserer Serverinfrastruktur, ein anderes der Druckerraum und in einem dritten ist die zur FMH Consulting gehörende Stellenvermittlung untergebracht. Im Kellergeschoss befinden sich drei von uns genutzte Archivräume, ein Kopierraum sowie die Keller zu den Wohnungen, die Heizung, Waschküche und: eine Dusche, die der seinerzeitige Generalsekretär, der regelmässig im nahen Dählhölzliwald joggen ging, in weiser Voraussicht einbauen liess und die auch heute von verschiedenen Mitarbeiterinnen und Mitarbeitern nach sportlicher Betätigung über die Mittagspause dankbar benutzt wird. Zum Haus gehören ein grosser Garten, der auf dem Dach der Autoeinstellhalle angelegt wurde (Abb. 4), und eine Reihe Aussenparkplätze. Mit Ausnahme von vier, speziell für
Besucher/innen des FMH-Generalsekretariates reservierten Parkplätzen sind alle übrigen an das Personal vermietet. Die Liegenschaft ist je zur Hälfte im Besitze der FMH und der FMH Services.

Von Anfang an war klar, dass wir einen Hauswart brauchten, der, wenn schon nicht vollamtlich angestellt, so doch im Hause selbst wohnte. Während vieler Jahre hatte die Familie Pfister die Hauswartfunktion inne. Hans Pfister, hauptberuflich bei der Ärztekasse tätig, die vor ihrem Umzug nach Gümligen im zweiten Stock unserer Liegenschaft eingemietet war, verfügte über ein grosses handwerkliches Geschick und einen ausgeprägten Geschäftssinn, der sich vor allem in Verhandlungen mit Lieferanten und Handwerkern bezahlt machte. In der Tradition eines guten Hauswartes hat er die Liegenschaft immer ein wenig als seine eigene betrachtet. Für die täglichen Putzarbeiten standen ihm Mitarbeiter einer externen Firma zur Verfügung, deren Arbeit er sehr aufmerksam beaufsichtigte. Als er sich entschloss, in den Ruhestand zu treten, war er es, der uns einen Nachfolger vorschlug.

Seit dem 1. Januar 1996 wohnt die Familie Manuel und Dorinda Gonzalez mit ihrem Sohn im «Ärztehaus». Sie kannten das Haus bestens, waren sie doch schon einige Jahre von Herrn Pfister mit der zweimal jährlich stattfindenden Fensterreinigung beauftragt worden. Auch Manuel Gonzalez geht tagsüber einer anderen beruflichen Tätigkeit nach. Jeden Abend machen er und seine Frau zusammen den «Putzkehr»: dazu gehören sämtliche Büros, das Treppenhaus, der Lift, Korridore, der Eingang, Parkplatz und Garten. Kleinere Reparaturen im Haus und an Maschinen sowie einfachere Gartenarbeiten werden von Manuel Gonzalez selbst ausgeführt. Grössere Bestellungen für Putz- und Verbrauchsmaterial sowie anstehende grössere Reparaturen

\section{Abbildung 2}

Durch diesen Eingang betreten Sie das FMH-Generalsekretariat.

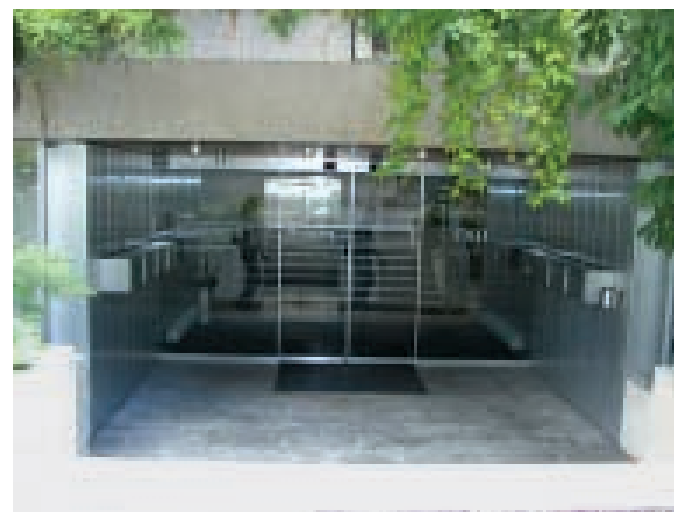


Abbildung 4

FMH-Generalsekretariat, Südseite mit Garten.

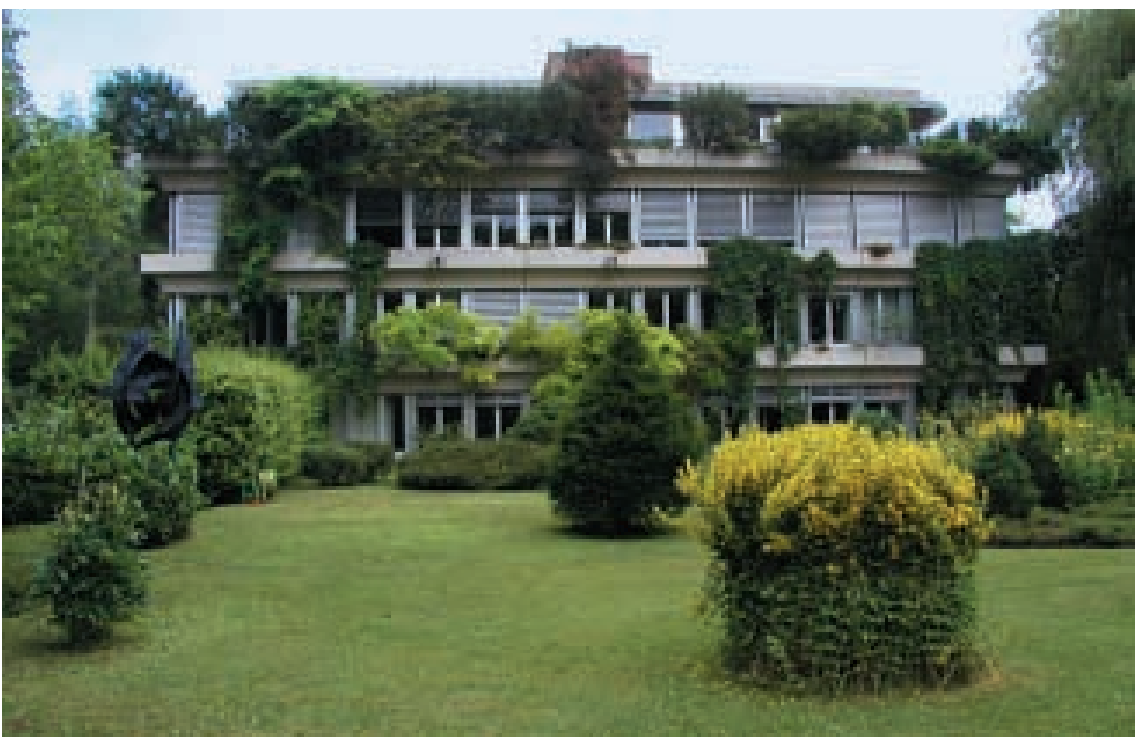

oder Sanierungen - bei einem fast 30jährigen Haus muss man naturgemäss mit solchen rechnen - werden mit der Leiterin Dienste besprochen. Diese holt die entsprechenden Offerten ein und gibt die Arbeiten in Auftrag. Dorinda Gonzalez sorgt dafür, dass bei angemeldeten Sitzungen die entsprechende Anzahl Gläser und
Getränke vorhanden sind, bereitet die Cafeteria vor, wenn ein Mittagessen oder sonst eine Verpflegung serviert wird, und besorgt anschliessend auch das Aufräumen und den Abwasch. Wenn in einer Abteilung «Not an der Frau» ist, übernimmt sie auch gerne einfachere Büroarbeiten, z. B. das Kopieren von Unterlagen oder das Verräumen von angeliefertem Papier. Manuel und Dorinda Gonzalez sind zu unseren guten Geistern im Haus geworden. Stets freundlich und guter Laune, haben sich zwischen unserem Hauswartehepaar und einigen Mitarbeiterinnen und Mitarbeitern, vor allem natürlich mit denjenigen, die häufig auch nach 17.00 Uhr noch im Büro arbeiten, sehr gute, ja freundschaftliche Beziehungen entwickelt. Auch für unsere beiden Mietparteien ist es beruhigend $\mathrm{zu}$ wissen, dass der Hauswart im Haus ist und in Notfällen auch nachts und an Wochenenden zu Hilfe gerufen werden kann. Ansprechpartnerin für die beiden Mieter im dritten Stock ist auch hier für grössere Reparaturen oder Fragen, die das Mietverhältnis betreffen, die Leiterin Dienste.

Im abgelaufenen Geschäftsjahr haben wir in einer ersten Etappe den Lift saniert; die zweite Sanierungsetappe, die zu einer massiven Reduktion der Stromkosten führen soll, ist im Laufe 2003 vorgesehen. Geplant ist für 2003 auch die Neugestaltung unserer Cafeteria.

\title{
Service de conciergerie/Immeuble FMH
}

\author{
Rapport d'activité 2002
}

Erika Flückiger

C'est le jeudi 14 mars 1974 que le projet «Démolition des bâtiments sis au 18, 18A et $18 \mathrm{~B}$ de l'Elfenstrasse et construction d'un immeuble administratif pour les médecins avec parking souterrain de 21 places» a été publié dans la Feuille officielle («Anzeiger») de la ville de Berne. Le même mois était diffusée une ébauche de nouveau plan de zone, résultant d'une motion approuvée à une large majorité - en automne 1972 - par le conseil municipal de la ville de Berne. Il s'agissait de freiner l'augmentation inquiétante des bureaux et autres places de travail tout en préservant et augmentant les espaces réservés à l'habitat.

Situé dans le quartier de Brunnadern, l'immeuble des médecins, siège du Secrétariat géné- ral de la FMH, est entouré d'ambassades et de consulats (fig. 2). Lors de la construction, il a fallu tenir compte des directives du plan de zone. C'est pourquoi on a construit deux grands appartements en attique au troisième étage. Dès le départ, un autre logement a été prévu pour les concierges. Dans les commentaires accompagnant la demande de permis de construire, on peut lire entre autres: «Le projet se caractérise par une parfaite intégration dans ce quartier de diplomates et de villas. Loin d'un banal immeuble de bureaux, il s'agit, d'un point de vue tant économique qu'humain, d'une véritable Domus medica dont l'esthétique est à la hauteur des élégantes demeures voisines. Les arbres et la verdure qui l'entourent sont préservés au mieux. 
Une statique et un plan des étages «en quadrillage», de même que le recours à des parois amovibles, permettent en tout temps de modifier la disposition des locaux.» (fig. 3)

$\mathrm{Au}$ cours des années, nous avons tiré parti à plusieurs reprises de cette structure modulable, notamment pour créer de nouveaux bureaux lors de déménagements internes ou de la restructuration de certains services. Mais cette possibilité de déplacer les parois sans trop de peine a aussi suscité de nouvelles envies: à l'heure actuelle, le rez-de-chaussée du Secrétariat général abrite une spacieuse salle de réunion équipée d'un rétroprojecteur, d'un «beamer» installé à demeure, d'un tableau à feuilles mobiles, d'un vidéo-téléviseur et de suffisamment de prises pour ordinateurs portables, salle utilisée tant par le Comité central que par les commissions de la FMH et les divers services du Secrétariat général. Toujours au rez-de-chaussée, on trouve une cafétéria fréquentée par le personnel pendant les pauses-café ou de midi et par le CC qui y déjeune lors de ses séances, la réception et la centrale téléphonique, de même que sept bureaux occupés par le Département de la formation prégraduée, postgraduée et continue (FPPC). Le premier étage comprend quatorze bureaux et l'appartement des concierges. Le deuxième renferme quant à lui seize bureaux, dont la salle où se trouvent nos serveurs et l'infrastructure informatique, le centre d'impression et le Bureau de placement rattaché à FMH Consulting. Au sous-sol enfin sont hébergés le chauffage, la buanderie, un local de photocopie, trois salles d'archives internes, les caves attribuées aux appartements, sans oublier une douche: installée sur la sage initiative du secrétaire général de l'époque qui faisait régulièrement du jogging dans la forêt du «Dählhölzli» toute proche, elle continue à être utilisée - et appréciée - par divers collaborateurs qui pratiquent un sport pendant la pause de midi. Enfin, font encore partie de l'immeuble un grand jardin aménagé sur le toit du parking souterrain (fig. 4) et une série de places de parc extérieures. Cellesci sont louées au personnel, à l'exception de quatre d'entre elles réservées aux visiteurs du Secrétariat général. L'immeuble de l'Elfenstrasse appartient à parts égales à la FMH et à FMH Services.

D'emblée, il est apparu clairement que nous avions besoin d'un concierge, engagé certes à temps partiel mais habitant sur place. Ce poste a été occupé pendant de nombreuses années par la famille Pfister. Travaillant pour la Caisse des médecins qui - avant son transfert à Gümligen louait le deuxième étage de notre immeuble, Hans Pfister possédait une grande dextérité manuelle et un sens aigu des affaires, qualités qui se sont révélées fort précieuses pour négocier avec les fournisseurs ou les artisans. Comme tous les bons concierges, il a toujours considéré cette propriété un peu comme la sienne. Ainsi, il super-

Figure 1

Dorinda et Manuel Gonzalez.

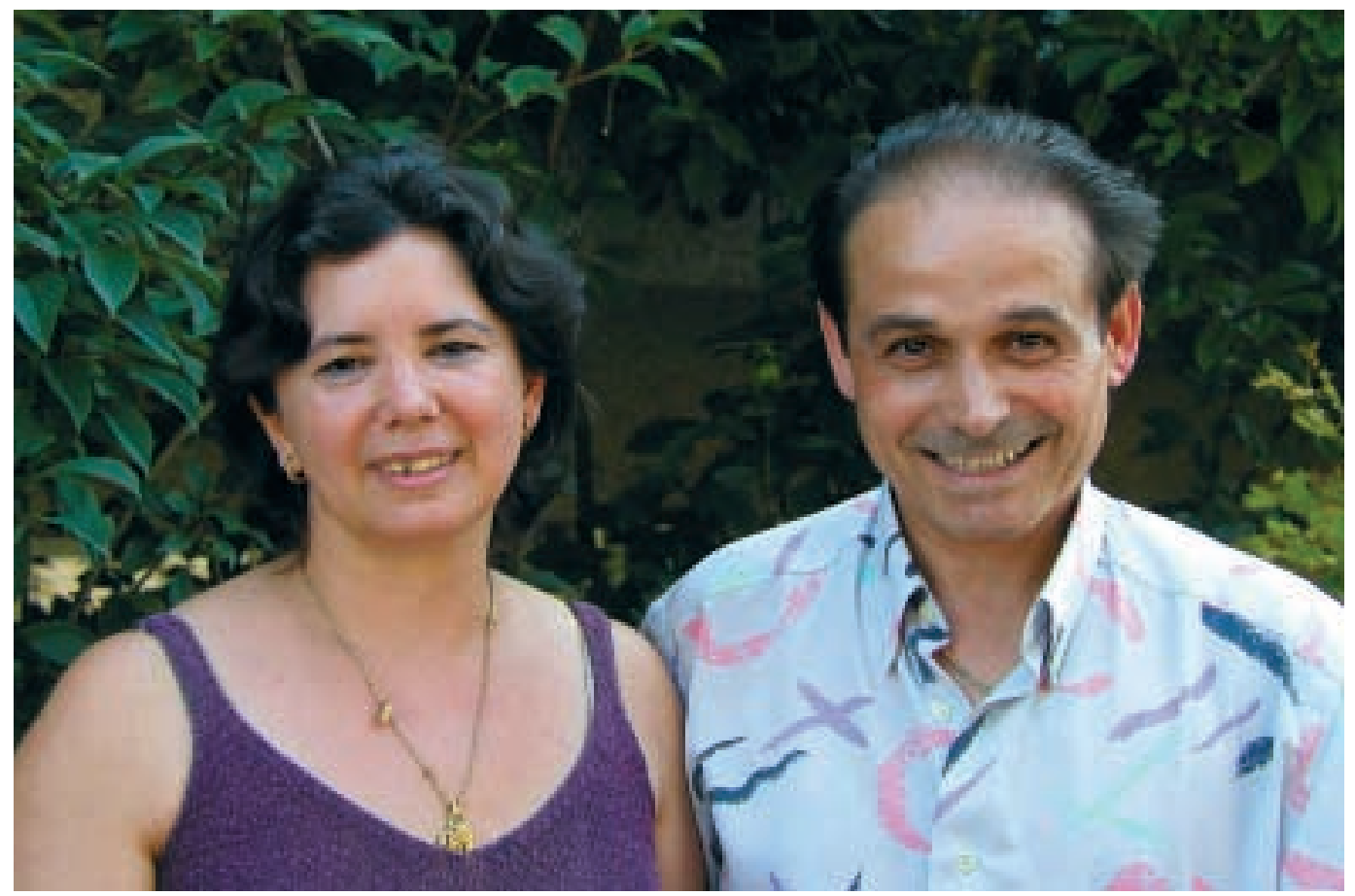


Figure 3

Secrétariat général de la FMH, vue nord/est.

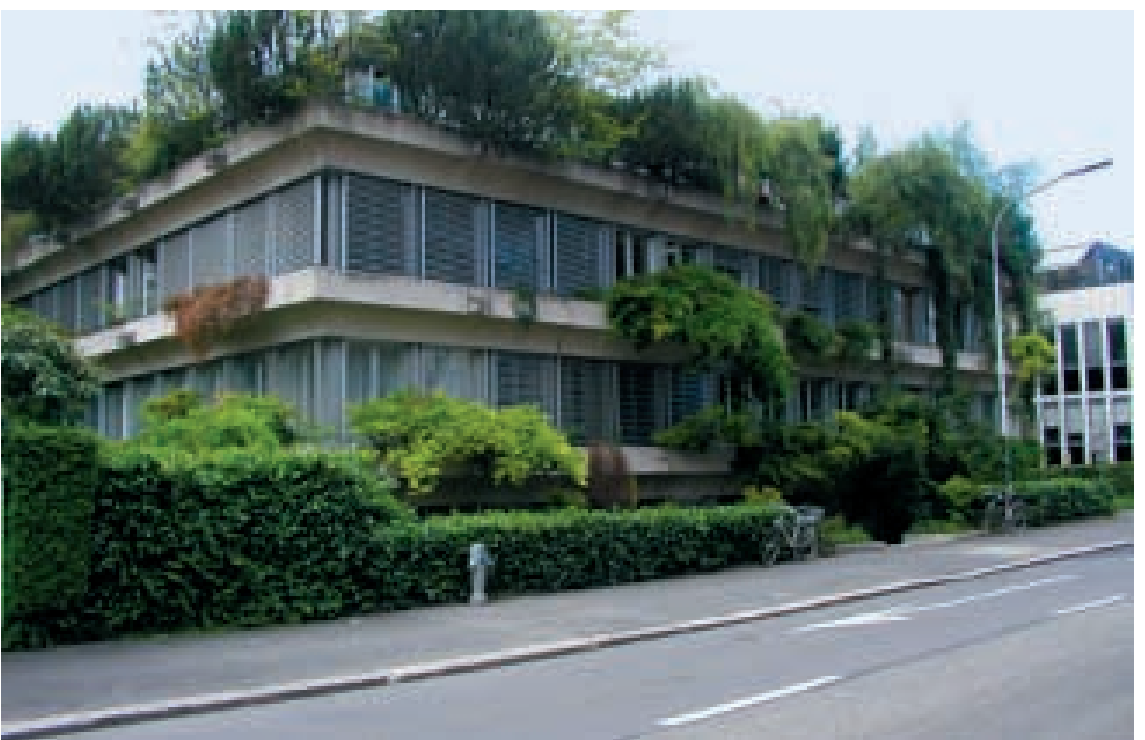

Figure 4

Secrétariat général de la FMH, vue sud avec jardin.

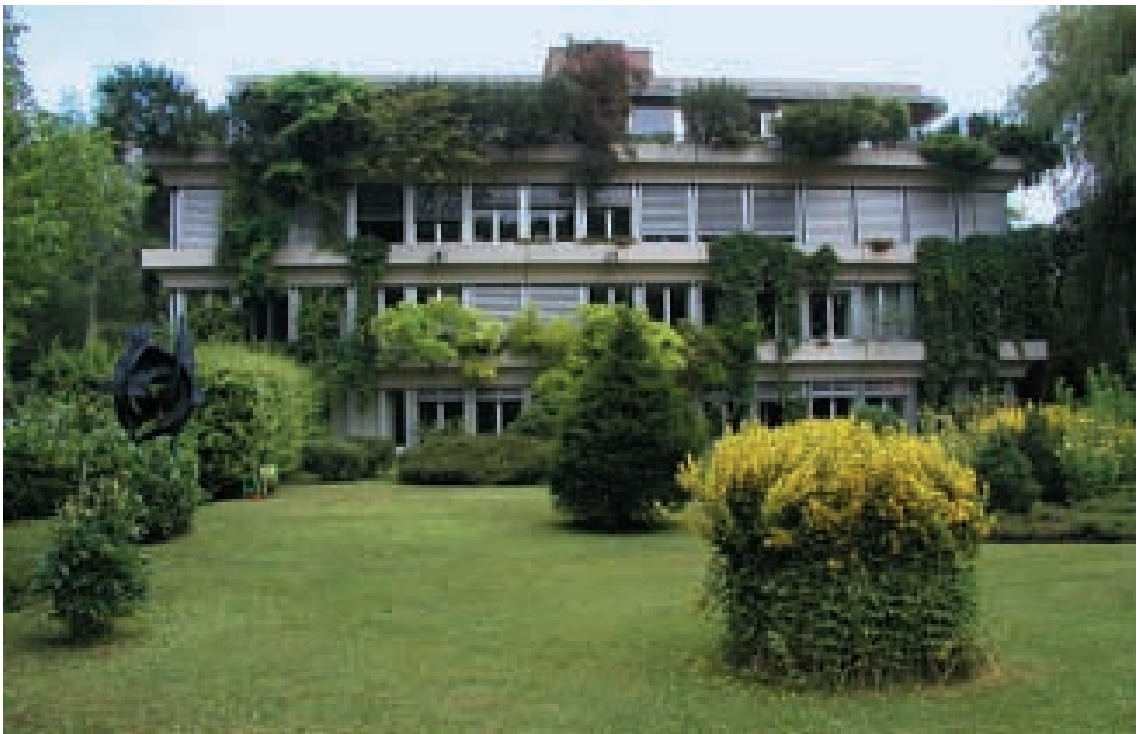

visait avec soin les travaux de nettoyage quotidiens, effectués par une entreprise extérieure. Et lorsqu'il décida de prendre sa retraite, c'est lui qui nous proposa son successeur.

Depuis le $1^{\text {er }}$ janvier 1996, l'appartement et la fonction de concierge sont occupés par Manuel et Dorinda Gonzalez accompagnés de leur fils. Ils connaissaient déjà bien l'immeuble pour en avoir nettoyé les fenêtres deux fois l'an sur mandat de M. Pfister! Comme son prédécesseur, Manuel Gonzalez exerce une autre activité professionnelle pendant la journée. Le soir, son épouse et lui-même accomplissent la tournée de nettoyage, qui englobe tous les bureaux, la cage d'escalier, l'ascenseur, les corridors, l'entrée et les places de parc. Les petites réparations dans l'immeuble ou aux machines sont exécutées par M. Gonzalez lui-même. Pour les grandes commandes de produits de nettoyage et de matériel d'usage courant, ainsi que pour les réparations ou rénovations plus importantes (inévitables dans un immeuble de presque trente ans), il consulte la responsable des services. Celle-ci demande alors un devis et procède à l'attribution externe des travaux. De son côté, Dorinda Gonzalez veille à ce qu'il y ait toujours suffisamment de verres et de boissons lors des réunions, prépare la cafétéria avant qu'on y serve un repas ou une collation et la remet en ordre après usage, lavage de la vaisselle y compris. En cas de surcharge de tel ou tel service, elle s'occupe aussi volontiers de travaux de bureau tels que la production de photocopies ou le rangement de papier venant d'être livré. Toujours aimables et de bonne humeur, Manuel et Dorinda Gonzalez sont en quelque sorte devenus les bons génies de la maison. Entre eux et certains collaborateurs, surtout ceux qui travaillent souvent après $17 \mathrm{~h} 00$, de véritables liens d'amitié se sont noués. Pour nos deux locataires du troisième étage, il est en outre rassurant de savoir que les concierges habitent sur place et peuvent être appelés à l'aide en cas d'urgence, même la nuit ou le week-end. Signalons encore que ces mêmes locataires s'adressent à la responsable des services pour les réparations importantes ou les questions touchant au bail à loyer.

Lors de l'exercice écoulé, une révision de l'ascenseur a été menée à bien. Une deuxième étape d'assainissement, prévue pour 2003, permettra de réduire considérablement les frais d'électricité. La rénovation de notre cafétéria figure également au programme de cette année.

Figure 2

L'entrée du Secrétariat général de la FMH.

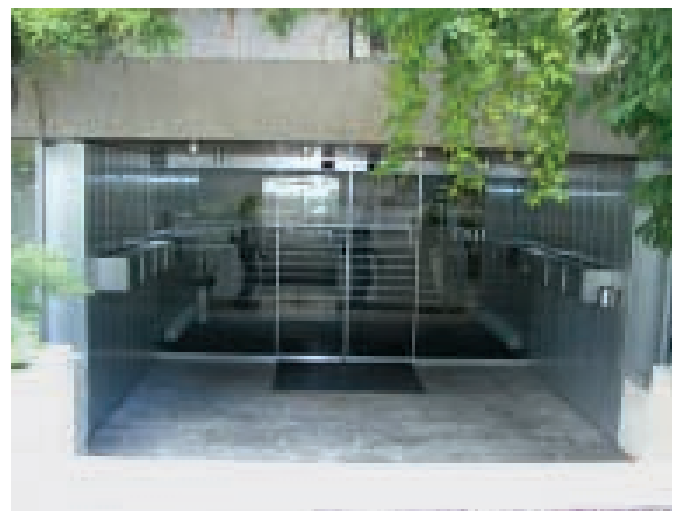

